\title{
TOPOLOGICAL SYMMETRY GROUPS OF COMPLETE GRAPHS IN THE 3-SPHERE
}

\author{
ERICA FLAPAN, RAMIN NAIMI, AND HARRY TAMVAKIS
}

\section{INTRODUCTION}

The topological symmetry group of an embedded graph in the 3-sphere was first defined by Jon Simon [Si] as a way of describing the symmetries of flexible molecules. The paper [FNPT] began to study the properties of these groups for arbitrary finite and connected embedded graphs. Our aim here is to determine the set of all orientation preserving topological symmetry groups for embeddings of complete graphs in $S^{3}$.

An embedded graph $\Gamma$ is a pair $(V, E)$ of sets of vertices $V$ and edges $E$ such that $V$ is a set of points in $S^{3}$, every edge is an embedded arc in $S^{3}$ between two vertices, and the interior of each edge contains no vertex and no point of any other edge. We shall abuse notation and say that $\Gamma$ is an embedding of its underlying abstract graph $\gamma$ to mean that $\Gamma$ is the image of an embedding of $\gamma$ in $S^{3}$.

We will be considering diffeomorphisms $g$ of $S^{3}$ which take an embedded graph $\Gamma$ to itself. We will use the notation $g:\left(S^{3}, \Gamma\right) \rightarrow\left(S^{3}, \Gamma\right)$ to mean not only that $g(\Gamma)=\Gamma$, but that $g(V)=V$ and $g(E)=E$ as well. Any such $g$ defines an automorphism of the underlying abstract graph $\gamma$; we shall say that this automorphism is induced by $g$. The topological symmetry group, $\operatorname{TSG}(\Gamma)$, is defined to be the subgroup of $\operatorname{Aut}(\gamma)$ consisting of those automorphisms which are induced by some diffeomorphism $g:\left(S^{3}, \Gamma\right) \rightarrow$ $\left(S^{3}, \Gamma\right)$. If we allow only orientation preserving diffeomorphisms of $S^{3}$, we obtain the orientation preserving topological symmetry group, $\operatorname{TSG}_{+}(\Gamma)$.

Topological symmetry groups of embeddings of the complete graph $K_{n}$ are interesting to consider because $K_{n}$ has the largest possible automorphism group of any graph with $n$ vertices. For every $n \leqslant 5$ it is not hard to find an embedding $\Gamma$ of $K_{n}$ with $\operatorname{TSG}(\Gamma)=S_{n}$ (the symmetric group). However, it was shown in $[\mathrm{F}]$ that any embedding $\Gamma$ of $K_{n}$ with $n \geqslant 6$, the cycle automorphism (1234) of $K_{n}$ cannot be induced by any diffeomorphism of $\left(S^{3}, \Gamma\right)$. Thus if $\Gamma$ is an embedding of $K_{n}$ with $n \geqslant 6$, then $\operatorname{TSG}(\Gamma)$ is a proper subgroup of $S_{n}$.

Other known examples include the trivial group which can be obtained for any $n \geqslant 4$ by letting $\Gamma$ be an embedding of $K_{n}$ which has non-homeomorphic

Date: April 27, 2004.

1991 Mathematics Subject Classification. 05C10, 57M15; 05C25.

The third author was supported in part by NSF Grant DMS-0296023. 
knots tied in every edge, and every dihedral group $D_{n}$ with $n \geqslant 7$ which was shown by Noda $[\mathrm{N}]$ to be TSG $(\Gamma)$ for a particular embedding $\Gamma$ of $K_{n}$.

For $n \leqslant 3$, it is not hard to see that each of the non-trivial subgroups of $\operatorname{Aut}\left(K_{n}\right)$ can be $\operatorname{TSG}_{+}(\Gamma)$ for some embedding $\Gamma$ of $K_{n}$. So we concentrate on the case where $n \geqslant 4$. In this case, Theorem 2 of [FNPT] shows that for any embedding $\Gamma$ of $K_{n}, \mathrm{TSG}_{+}(\Gamma)$ is isomorphic to a finite subgroup of the group Diff $+\left(S^{3}\right)$ of orientation preserving diffeomorphisms of $S^{3}$. Our task is therefore to determine which of the finite subgroups of $\operatorname{Diff}_{+}\left(S^{3}\right)$ are actually possible for embeddings of complete graphs. This is the content of our main result.

Theorem 1. A finite group $H$ is $\mathrm{TSG}_{+}(\Gamma)$ for an embedding $\Gamma$ of some $K_{n}$ if and only if $H$ is isomorphic to a finite subgroup of either $S O(3)$ or $D_{m} \times D_{m}$ for some odd $m$.

We begin by proving in Proposition 1 that we only need to consider those embeddings $\Gamma$ of $K_{n}$ such that $\mathrm{TSG}_{+}(\Gamma)$ is induced by an isomorphic group of orientation preserving isometries of $S^{3}$. Furthermore, in Lemma 2 we prove that for every such embedding $\Gamma$ of a complete graph, the involutions in $T S G_{+}(\Gamma)$ must satisfy a key condition. The proof that the groups listed in Theorem 1 are the only ones possible proceeds in Section 3 by considering all finite subgroups $G$ of $S O(4)$ that satisfy this involution condition and examining two separate cases, according to whether or not $G$ preserves some Seifert fibration of $S^{3}$. In Section 4, we prove that all of the groups listed in Theorem 1 can actually occur as $T S G_{+}(\Gamma)$ for some embedding $\Gamma$ of a complete graph $K_{n}$.

The first and third authors began collaborating on this project in the fall of 2000 during a visit to the Institut des Hautes Études Scientifiques. It is a pleasure to thank the Institut for its hospitality. The second author wishes to thank the California Institute of Technology for its hospitality during his sabbatical in the spring of 2002 .

\section{Preliminary Results}

Let $\Gamma$ be a graph embedded in $S^{3}$ and let $H$ be a subgroup of $\operatorname{TSG}_{+}(\Gamma)$. Suppose $G$ is a group of orientation preserving diffeomorphisms of $\left(S^{3}, \Gamma\right)$ such that for every $a \in H$ there is a $g_{a} \in G$ which induces $a$ on $\Gamma$, and every $g \in G$ induces some element of $H$ on $\Gamma$. Then we say that $G$ induces $H$ on $\Gamma$. A finite group $H$ is said to be realizable by an embedded graph $\Gamma$ if $H \cong \mathrm{TSG}_{+}(\Gamma)$.

We begin by recalling a result proved in [FNPT].

Lemma 1 ([FNPT]). Let $\Omega$ be an embedded 3-connected graph with $H=$ $\mathrm{TSG}_{+}(\Omega)$. Then $\Omega$ can be re-embedded as $\Delta$ such that $H \leqslant \mathrm{TSG}_{+}(\Delta)$ and $\mathrm{TSG}_{+}(\Delta)$ is induced by an isomorphic subgroup of $\operatorname{Diff}_{+}\left(S^{3}\right)$.

This lemma tells us that to classify $\operatorname{TSG}_{+}(\Gamma)$ for embeddings of $K_{n}$ with $n \geq 4$, we only need to consider embeddings $\Gamma$ where $\operatorname{TSG}_{+}(\Gamma)$ is induced 
by an isomorphic finite subgroup of Diff $+\left(S^{3}\right)$. In analyzing finite order diffeomorphisms of $S^{3}$, it is helpful to look at their fixed point sets. Smith characterized the fixed point sets of finite order homeomorphisms of homology spheres [Sm]. In particular, he showed that the fixed point set, fix $(g)$, of any non-trivial orientation preserving finite order homeomorphism $g$ of $S^{3}$ is either empty or homeomorphic to $S^{1}$. This result will be a useful tool for us, which we will refer to as Smith theory. Note that if $g$ is an orientation preserving isometry of $S^{3}$, then this result is true even without making use of Smith's work. However, for consistency we will nonetheless refer to this as Smith theory. We shall use Smith theory to prove that if $\Gamma$ is an embedding of $K_{n}$, then any involution in $\mathrm{TSG}_{+}(\Gamma)$ satisfies a key condition.

Lemma 2. Let $\Gamma$ be an embedding of $K_{n}$ with $n \geq 4$, and let $G$ be a finite subgroup of $\operatorname{Diff}_{+}\left(S^{3}\right)$ which leaves $\Gamma$ setwise invariant. For every involution $g \in G$, fix $(g) \cong S^{1}$, and no $h \in G$ distinct from $g$ satisfies $\operatorname{fix}(h)=\operatorname{fix}(g)$.

Proof. Let $g \in G$ be an involution. If $g$ fixes any point of $S^{3}$, then by Smith Theory fix $(g) \cong S^{1}$. Since $g$ has finite order and $g(\Gamma)=\Gamma$, if $g$ fixes every vertex of $\Gamma$ then fix $(g)$ would contain $\Gamma$. As $n \geq 4$, this would contradict Smith theory, and hence there is some vertex $v$ which is not fixed by $g$. Let $e$ be the edge connecting $v$ and $g(v)$. Then $g(e)=e$, and hence $g$ fixes precisely one point of $e$. Thus fix $(g) \cong S^{1}$ and $g$ fixes precisely one point of each edge whose vertices are interchanged by $g$. It follows that every vertex of $\Gamma$ is either contained in $\operatorname{fix}(g)$ or is an endpoint of an edge meeting fix $(g)$.

Now suppose that $h \in G$ such that $\operatorname{fix}(h)=\operatorname{fix}(g)$. Let $v$ be a vertex of $\Gamma$. If $v \in \operatorname{fix}(g)$, then $v$ is fixed by both $g$ and $h$. Suppose that $v \notin \operatorname{fix}(g)$. Then $v$ is a vertex of an edge $e$ meeting $\operatorname{fix}(g)=\operatorname{fix}(h)$. Thus both $g$ and $h$ interchange the endpoints of $e$. Hence $g$ and $h$ must induce precisely the same automorphism on the vertices of $\Gamma$. Since $g$ and $h$ both have finite order, it follows that $g|\Gamma=h| \Gamma$, and hence $h g^{-1}$ fixes $\Gamma$ pointwise. Now since $n>3$, it follows from Smith theory that $h=g$.

We will use these lemmas as follows to prove that we only need to consider isometries of $S^{3}$ rather than arbitrary diffeomorphisms.

Proposition 1. Let $\Omega$ be an embedding of some $K_{n}$, and let $H=\operatorname{TSG}_{+}(\Omega)$. Then either $H$ is a cyclic group of odd order or $\Omega$ can be re-embedded as $\Gamma$ such that $H \leqslant \mathrm{TSG}_{+}(\Gamma)$ and $\mathrm{TSG}_{+}(\Gamma)$ is induced by an isomorphic subgroup of $S O(4)$.

Proof. First suppose that $n \leqslant 3$. Let $\Gamma$ be a symmetric planar embedding of $K_{n}$. Then Aut $(\Gamma)=\mathrm{TSG}_{+}(\Gamma)$ is induced by an isomorphic subgroup of $S O(4)$.

Now assume that $n>3$ and $H$ is not a cyclic group of odd order. Since $K_{n}$ is 3 -connected, it follows from Lemma 1 that $\Omega$ can be re-embedded as $\Delta$ such that $H \leqslant \operatorname{TSG}_{+}(\Delta)$ and $\operatorname{TSG}_{+}(\Delta)$ is induced by an isomorphic finite subgroup $F$ of Diff $+\left(S^{3}\right)$. Suppose $F$ acts freely on $S^{3}$. By Lemma $2,|F|$ must be odd. However, cyclic groups are the only odd order groups which 
can act freely on $S^{3}$ (see [O, 6.2.1 and 6.3 i-iii]); hence $F$ cannot act freely on $S^{3}$. Thus by Smith theory, $F$ contains some element whose fixed point set is homeomorphic to $S^{1}$. Now by Thurston's Orbifold Theorem [BP, $\mathrm{BLP}, \mathrm{CHK}], F$ is conjugate to a subgroup $G$ of $S O(4)$ by an orientation preserving diffeomorphism $f$. Let $\Gamma=f(\Delta)$, then $G$ induces $\operatorname{TSG}_{+}(\Gamma)$ and $G \cong \mathrm{TSG}_{+}(\Gamma) \cong \mathrm{TSG}_{+}(\Delta)$.

Let $H=\mathrm{TSG}_{+}(\Gamma)$ for some embedding $\Gamma$ of a complete graph. By Proposition 1, we can assume that either $H$ is a cyclic group of odd order or there is an isomorphic finite subgroup of $S O(4)$ which induces $H$ on $\Gamma$. If $H$ is cyclic, then it is one of the groups listed in Theorem 1. So we assume instead that $H$ is induced by an isomorphic finite subgroup of $S O(4)$. This assumption, together with the conclusion of Lemma 2, are all that we will use about topological symmetry groups in order to prove that $H$ is isomorphic to one of the groups listed in Theorem 1. Thus, rather than continuing to work with $\operatorname{TSG}_{+}(\Gamma)$, we shall now work with arbitrary finite subgroups of $S O(4)$ which satisfy the conclusion of Lemma 2. Since this conclusion will play an important role in our proofs, we shall henceforth refer to it as the Involution Condition.

Definition 1. A finite subgroup $G$ of $\operatorname{Diff}_{+}\left(S^{3}\right)$ satisfies the Involution Condition if for every involution $g \in G$, fix $(g) \cong S^{1}$, and no $h \in G$ distinct from $g$ satisfies $\operatorname{fix}(h)=\operatorname{fix}(g)$.

Observe that if $g$ and $h$ are any pair of involutions in Diff $+\left(S^{3}\right)$ with fix $(g) \cong S^{1}$ and fix $(g)=\operatorname{fix}(h)$, then certainly $g=h$. Thus the point of the Involution Condition is that if $g \in G$ is an involution then fix $(g) \cong S^{1}$ and if $h \in G$ with $\operatorname{order}(h)>2$, then $\operatorname{fix}(h) \neq \operatorname{fix}(g)$. In particular, if $h \in G$ which has even order greater than two, then $h$ must be fixed point free.

\section{Subgroups of $S O(4)$ that satisfy the Involution Condition}

Let $G$ be a finite subgroup of $S O(4)$ which satisfies the Involution Condition. We will prove in this section that $G$ is isomorphic to a finite subgroup of either $S O(3)$ or of $D_{m} \times D_{m}$ with $m$ odd.

We consider two cases according to whether or not $G$ preserves some Seifert fibration of $S^{3}$. First we assume that $G$ does preserve a Seifert fibration of $S^{3}$. We will use the following terminology.

Notation. Let $\pi: G \rightarrow G_{b}$ denote the projection map from $G$ to the group $G_{b}$ acting on the base space of the fibration. Let $N=\operatorname{ker}(\pi)$, let $G_{b}^{+}$denote the orientation preserving subgroup of $G_{b}$, and let $G^{+}=\pi^{-1}\left(G_{b}^{+}\right)$. We thus have the following commutative diagram with exact rows of subgroups of $S O(4)$. 


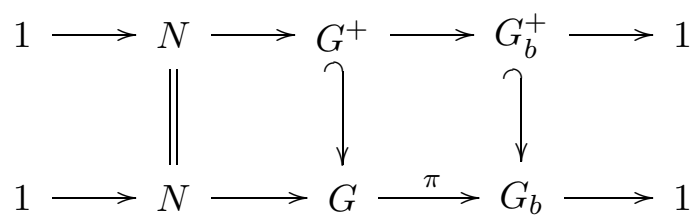

We choose the metric on the base space $S^{2}$ so that $G_{b}$ is a group of isometries. Hence $G_{b}^{+}$is a finite subgroup of $S O(3)$. It is now easy to see that if $g \in G^{+}-N$, then there are precisely two fibers of $S^{3}$ which are each setwise invariant under $g$. Also, since $S^{3}$ and the base space are each orientable, every $h \in N$ preserves the orientation of every fiber. Thus since $N$ is determined by its action on a non-exceptional fiber, $N$ is a cyclic group.

We begin by using Smith theory and the Involution Condition to prove three elementary facts.

Fact 1. Let $g \in G$. Then the following are equivalent.

1) $g$ reverses the orientation of some fiber.

2) $g$ reverses the orientation of a circle of fibers, and leaves no other fiber setwise invariant.

3) $\pi(g)$ is a reflection.

4) $g \notin G^{+}$and $\operatorname{order}(g)=2$.

Proof. We prove $1 \Rightarrow 2 \Rightarrow 3 \Rightarrow 2 \Rightarrow 1$ and $3 \Rightarrow 4 \Rightarrow 3$. Suppose that $g$ reverses the orientation of some fiber $l$. Then $g$ fixes precisely two points on $l$, and hence by Smith theory $\operatorname{fix}(g) \cong S^{1}$. Thus there must be a circle of fibers which are reversed by $g$, and hence $\pi(g)$ pointwise fixes a circle $C$ which is the projection of these fibers. Since $g \notin N$ and $\pi(g)$ is an isometry of $S^{2}$, fix $(\pi(g))=C$. Hence the fibers in this circle are the only ones which are setwise invariant under $g$, and $\pi(g)$ must be a reflection.

Now let $g \in G$ such that $g^{\prime}=\pi(g)$ is a reflection of $S^{2}$. Now fix $\left(g^{\prime}\right)$ lifts to a fibered torus $T$ in $S^{3}$ such that $g$ takes each fiber on $T$ to itself and interchanges the fibers in the two complementary solid tori. It follows that $g$ reverses the orientation of the circle of fibers making up $T$ and leaves no other fiber setwise invariant. Furthermore, $g^{2}$ pointwise fixes each fiber of $T$. Thus, by Smith theory, $\operatorname{order}(g)=2$. Also since $\pi(g)$ is a reflection, $g \notin G^{+}$.

Finally, let $g \notin G^{+}$with order $(g)=2$. By the Involution Condition, fix $(g) \cong S^{1}$. Thus $\pi(g)$ has non-empty fixed point set. Since $\pi(g) \in S O(3)$ and is orientation reversing, $\pi(g)$ must be a reflection.

Fact 2. Every element of $G^{+}$commutes with every element of $N$.

Proof. Let $g \in G^{+}$. Then $\pi(g)$ is a rotation, hence there are fibers $k_{1}$ and $k_{2}$ such that $g\left(k_{i}\right)=k_{i}$. By Fact $1, g$ preserves the orientation of each $k_{i}$. Let $h \in N$. Since $h$ also preserves the orientation of each $k_{i}, g h g^{-1} h^{-1} \mid k_{i}$ is 
the identity for both $i=1$ and $i=2$. Hence it follows from Smith theory that $g h g^{-1} h^{-1}$ is the identity.

Fact 3. Every involution in $G^{+}$pointwise fixes a fiber, and if there are no exceptional fibers then $|N|$ is odd.

Proof. Let $g \in G^{+}$be an involution; by the Involution Condition, fix $(g) \cong$ $S^{1}$. Since $g$ is fiber preserving, if $l$ is a fiber which intersects fix $(g)$ then either $g$ fixes two points of $l$ or $l=\operatorname{fix}(g)$. If $g$ fixes two points of $l$, then $g$ reverses the orientation of $l$, and hence $g \notin G^{+}$by Fact 1 . Thus fix $(g)$ must be a fiber.

Finally, suppose $N$ contains an involution $g$; then fix $(g)$ must be an exceptional fiber. Thus if there are no such fibers, then $|N|$ is odd.

Notation. Let $T, O$, and $D$ denote the groups of orientation preserving symmetries of the regular tetrahedron, octahedron, and dodecahedron, respectively.

Lemma 3. There exist fibers $A$ and $B$, which are the only possible exceptional fibers, and $\{A, B\}$ is setwise invariant under $G$. Furthermore, let $J$ denote the subgroup of $G$ which takes each of $A$ and $B$ to itself, preserving the orientation of each. If $G$ contains an involution which interchanges $A$ and $B$, then $|J|$ is odd.

Proof. We begin with some trivial cases. If the fibration has two exceptional fibers, we are done by choosing the exceptional fibers to be $A$ and $B$. So we assume there is at most one exceptional fiber. If $G_{b}$ is trivial we are done by choosing $A$ or $B$ to be exceptional fibers if possible, and otherwise to be any fibers. Suppose $G_{b}$ is non-trivial but $G_{b}^{+}$is trivial. Then $G_{b} \cong \mathbb{Z}_{2}$ with some generator $\gamma$. If there is one exceptional fiber, let it be $A$. In this case, $\operatorname{fix}(\gamma)$ is non-empty since it contains the projection of $A$, and hence $\gamma$ is a reflection. So we are done by letting $B$ be any fiber which projects to a different point of $\operatorname{fix}(\gamma)$. Suppose there are no exceptional fibers, let $A$ and $B$ be any pair of fibers which project to a pair of points that are interchanged by $\gamma$. Since $G_{b}^{+}$is trivial, $J=N$ by Fact 1 , and $|N|$ is odd by Fact 3. So again we are done.

We may therefore assume that there is at most one exceptional fiber and $G_{b}^{+}$is non-trivial. We begin by showing that there cannot be a pair of involutions $f_{1} \in G_{b}^{+}$and $f_{2} \in G_{b}$ and distinct points $\left\{a_{1}, b_{1}, a_{2}, b_{2}\right\}$ in $S^{2}$ such that for $i \neq j, f_{i}$ fixes $a_{i}$ and $b_{i}$ and interchanges $a_{j}$ and $b_{j}$.

Suppose that there is such a pair of involutions $f_{1}$ and $f_{2}$. Let $A_{1}, A_{2}$, $B_{1}$ and $B_{2}$ be the fibers which project down to the points $a_{1}, a_{2}, b_{1}$ and $b_{2}$ respectively. For each $i$, let $g_{i} \in G$ be such that $\pi\left(g_{i}\right)=f_{i}$. It follows from our conditions on $f_{1}$ and $f_{2}$ that $g_{i}\left(A_{i}\right)=A_{i}, g_{i}\left(B_{i}\right)=B_{i}$, and for $j \neq i$, $g_{i}$ interchanges $A_{j}$ and $B_{j}$. Also, since $f_{1}$ is a non-trivial element of $G_{b}^{+}, a_{1}$ and $b_{1}$ are the only points of $S^{2}$ fixed by $f_{1}$. Thus $A_{1}$ and $B_{1}$ are the only fibers which are setwise invariant under $g_{1}$. Now since $g_{2}$ interchanges $A_{1}$ 
and $B_{1}$, there can be no exceptional fibers. So by Fact $3,|N|$ is odd. Let $n=\operatorname{order}\left(g_{1}\right)$; then $n$ is even since $f_{1}$ is an involution. So we let $h_{1}=\left(g_{1}\right)^{n / 2}$. Since $h_{1}$ is an involution, $h_{1} \notin N$. Hence the fibers $A_{1}$ and $B_{1}$ are the only fibers which are left setwise invariant by $h_{1}$. In particular, $h_{1}$ interchanges $A_{2}$ and $B_{2}$. Also since $h_{1} \in G^{+}$, it follows from Fact 3 that fix $\left(h_{1}\right)$ is a fiber. So without loss of generality, we can assume that fix $\left(h_{1}\right)=A_{1}$, and $\operatorname{order}\left(h_{1} \mid B_{1}\right)=2$.

Let $\alpha=\left(g_{2} h_{1}\right)^{2}$. Then $\alpha \in G^{+}$and $\alpha\left(A_{i}\right)=A_{i}$ and $\alpha\left(B_{i}\right)=B_{i}$ for each $i$. It follows that $\alpha \in N$. Since fix $\left(h_{1}\right)=A_{1}$ and $g_{2}$ interchanges $A_{1}$ and $B_{1}$, $\alpha\left|B_{1}=h_{1}\right| B_{1}$. It follows that $\operatorname{order}\left(\alpha \mid B_{1}\right)=2$, and hence $\alpha$ has even order. But this is a contradiction, since $|N|$ is odd.

It follows that the involutions $f_{1}$ and $f_{2}$ cannot simultaneously exist. Each of the groups $T, O$, and $D$, as well as the dihedral group $D_{r}$ where $r$ is even, contains such a pair of involutions $f_{1}$ and $f_{2}$. Hence either $G_{b}^{+}$is cyclic or $G_{b}^{+}=D_{r}$ where $r$ is odd. Thus we can define $J_{b}$ to be the largest cyclic subgroup of $G_{b}^{+}$. Since we are assuming that $G_{b}^{+}$is non-trivial, $J_{b}$ is a nontrivial cyclic group. Hence $J_{b}$ fixes precisely two points $x$ and $y$ of $S^{2}$. Now for every $\gamma \in G_{b}^{+}, \gamma \in J_{b}$ if and only if $\gamma$ fixes $x$ and $y$.

Let $\gamma \in G_{b}$. We will see as follows that $\gamma(\{x, y\})=\{x, y\}$. By hypothesis, there is some $\alpha \in J_{b}$ which is non-trivial. Now $\gamma^{-1} \alpha \gamma \in G_{b}^{+}$, and since either $G_{b}^{+}=J_{b}$ or $G_{b}^{+}$is dihedral, $\gamma^{-1} \alpha \gamma \in J_{b}$. Thus $\gamma^{-1} \alpha \gamma$ fixes both $x$ and $y$. This implies that $\alpha(\gamma(x))=\gamma(x)$ and $\alpha(\gamma(y))=\gamma(y)$. However, since $\alpha$ is a non-trivial element of $J_{b}, x$ and $y$ are the only points of $S^{2}$ fixed by $\alpha$; hence $\{\gamma(x), \gamma(y)\}=\{x, y\}$. Thus $\gamma(\{x, y\})=\{x, y\}$. Furthermore, there is a simple closed curve $C$ in $S^{2}-\{x, y\}$ which is setwise invariant under $G_{b}$.

Let $A$ and $B$ be the fibers that project to $x$ and $y$. Then $g(\{A, B\})=$ $\{A, B\}$, and since $J_{b}$ is non-trivial, no fiber other than $A$ and $B$ can be exceptional. Observe that, since $J$ preserves the orientations of $A$ and $B$, by Fact $1, J \leqslant G^{+}$and hence $\pi(J)=J_{b}$.

Now suppose there is some involution $g \in G$ which interchanges $A$ and $B$. Then there are no exceptional fibers and hence by Fact $3,|N|$ is odd. Now $\pi(g)$ interchanges $x$ and $y$ and fixes a pair $\{a, b\}$ of antipodal points on $C$. If $J_{b}$ contained an involution $f_{1}$, then $f_{1}$ would interchange $a$ and $b$. Hence the pair of involutions $f_{1}$ and $\pi(g)$ would violate our condition on pairs of involutions. Hence $\left|J_{b}\right|$ is odd, and since $|N|$ is odd, $|J|$ must be odd.

Notation. Let $A, B$, and $J$ be given by Lemma 3 , let $J_{b}=\pi(J)$, and let $x$ and $y$ denote the projections of $A$ and $B$. Let $C$ denote the unique simple closed curve on $S^{2}-\{x, y\}$ which is setwise invariant under $G_{b}$

Now we consider the possibilities for $J$.

Lemma 4. $J$ is cyclic or $J=\mathbb{Z}_{q} \times \mathbb{Z}_{r}$ with qr odd.

Proof. Since $J_{b}=\pi(J), J_{b}$ is the subgroup of $G_{b}^{+}$consisting of those elements which fix both $x$ and $y$. Hence $J_{b}$ is cyclic. Let $\alpha$ be a generator of the cyclic group $N$ and let $\beta \in J$ such that $\pi(\beta)$ is a generator of $J_{b}$. Then $\alpha$ 
and $\beta$ generate $J$. Since $\alpha$ and $\beta$ both preserve the orientation of $A$ and $B, \alpha \beta \alpha^{-1} \beta^{-1} \mid A$ and $\alpha \beta \alpha^{-1} \beta^{-1} \mid B$ are both the identity. Hence by Smith theory, $\alpha \beta \alpha^{-1} \beta^{-1}$ is the identity. Thus $J$ is abelian, and hence $J=\mathbb{Z}_{q} \times \mathbb{Z}_{r}$, for soem $q$ and $r$.

Suppose $J=\mathbb{Z}_{q} \times \mathbb{Z}_{r}$ is not cyclic and $q r$ is even. Then $J$ contains an involution $\gamma$, and by Fact $3, \operatorname{fix}(\gamma)$ is a fiber. If $\gamma \in N$, then $\operatorname{fix}(\gamma)$ is an exceptional fiber. Since $A$ and $B$ are the only possible exceptional fibers, fix $(\gamma)$ is either $A$ or $B$. On the other hand, if $\gamma \notin N$, then $A$ and $B$ are the only fibers which are setwise invariant under $\gamma$. Hence again $\operatorname{fix}(\gamma)$ is either $A$ or $B$. So without loss of generality, in either case we assume that $\operatorname{fix}(\gamma)=A$. Now by the Involution Condition, no other non-trivial element of $G$ fixes $A$ pointwise.

Suppose that $\delta \in J$ also has order 2 and $\delta \neq \gamma$. Then $\operatorname{fix}(\delta) \neq A$. Hence by the above argument, $\operatorname{fix}(\delta)=B$ and no other non-trivial element of $G$ fixes $B$ pointwise. Now since $J$ is abelian, $\gamma \delta$ also has order 2, and thus again by the above argument $\gamma \delta$ pointwise fixes either $A$ or $B$. As this is impossible, we conclude that $\gamma$ is the only involution in $J$.

Since $J=\mathbb{Z}_{q} \times \mathbb{Z}_{r}$ is not cyclic, $\operatorname{gcd}(q, r) \neq 1$. Now since $q r$ is even, and $J$ contains only one involution, without loss of generality, $q$ is odd and $r$ is even. Thus, there exists an odd prime $p$ such that $p \mid q$ and $p \mid \frac{r}{2}$. Choose generators $g_{1}$ and $g_{2}$ for $J$ such that $\operatorname{order}\left(g_{1}\right)=q$, order $\left(g_{2}\right)=r$, and the subgroups generated by $g_{1}$ and $g_{2}$ have trivial intersection. By definition of $J, A$ is setwise invariant under both $g_{1}$ and $g_{2}$. Since $\gamma$ is the only non-trivial element of $G$ that fixes $A$ pointwise, $\operatorname{order}\left(g_{1} \mid A\right)=q$ and $\operatorname{order}\left(g_{2} \mid A\right)=r / 2$. Let $i=q / p$, then $\operatorname{order}\left(g_{1}^{i} \mid A\right)=p$. Let $j=r /(2 p)$, then $\operatorname{order}\left(g_{2}^{j} \mid A\right)=p$. It follows that for some $k<p, g_{1}^{i k}\left|A=g_{2}^{j}\right| A$. But $g_{1}^{i k} \neq g_{2}^{j}$, since the groups generated by $g_{1}$ and $g_{2}$ have trivial intersection. Now since $g_{1}^{i k} g_{2}^{-j}$ is a nontrivial element of $G$ which fixes $A$ pointwise, $g_{1}^{i k} g_{2}^{-j}$ must be the involution $\gamma$. But this contradicts the fact that $\operatorname{order}\left(g_{1}^{i k} g_{2}^{-j}\right)$ divides $p$. Thus, if $J$ is not cyclic, then $q r$ is odd.

Lemma 5. If $G \neq J$, then we can choose an involution $\phi_{1}$ which interchanges $A$ and $B$ and/or an involution $\phi_{2}$ which reverses the orientation of $A$ and $B$ such that all of the following conditions hold:

1) $G$ is generated by $J$ together with whichever of $\phi_{1}$ and $\phi_{2}$ exists.

2) If both $\phi_{1}$ and $\phi_{2}$ exist, then they commute.

3) If $\phi_{2}$ exists, then for every $g \in J, \phi_{2} g \phi_{2}=g^{-1}$.

4) If $\phi_{1}$ exists, then we can choose generators $g_{1}$ and $g_{2}$ for $J$ such that $\phi_{1} g_{1} \phi_{1}=g_{1}^{-1}$ and $\phi_{1} g_{2} \phi_{1}=g_{2}$.

Proof. Since $G \neq J$, either $G$ contains an element $\phi$ which interchanges $A$ and $B$, or $G$ contains an element $\psi$ which reverses the orientation of both $A$ and $B$.

Suppose that $G$ contains an element $\phi$ which interchanges $A$ and $B$. Then there are no exceptional fibers. Hence by Fact $3,|N|$ is odd. Let $\phi_{1}$ denote $\phi$ to an appropriate odd power so that $\operatorname{order}\left(\phi_{1}\right)=2^{n}$. Then $\phi_{1}$ interchanges 
$A$ and $B$. Suppose that $n>1$. Let $\alpha=\left(\phi_{1}\right)^{2^{n-1}}$. Then $\alpha \in J$ and $\operatorname{order}(\alpha)=2$. By Fact 3 , $\operatorname{fix}(\alpha)$ is a fiber. Since $|N|$ is odd, $\alpha \notin N$. Thus $A$ and $B$ are the only fibers which are setwise invariant under $\alpha$. So, without loss of generality we can assume $\operatorname{fix}(\alpha)=A$. Since $\phi_{1}$ interchanges $A$ and $B, \phi_{1} \alpha \phi_{1}^{-1} \mid B$ is the identity. But $\phi_{1} \alpha \phi_{1}^{-1}=\alpha$, because $\alpha=\left(\phi_{1}\right)^{2^{n-1}}$. By Smith theory, $\alpha$ cannot pointwise fix both $A$ and $B$. Thus $n=1$, and $\phi_{1}$ is an involution.

If $G$ contains an element $\psi$ which reverses the orientation of both $A$ and $B$, then by Fact $1, \psi \notin G^{+}$and $\psi$ is an involution. So let $\phi_{2}=\psi$. Now $G$ is generated by $J$ together with the involutions $\phi_{1}$ and/or $\phi_{2}$, and hence we have proved Property (1).

To prove Property (2), suppose that $G$ contains an involution $\phi$ which interchanges $A$ and $B$ and an involution $\phi_{2}$ which reverses the orientation of both $A$ and $B$. By Fact $1, \phi_{2} \notin G^{+}$. We choose $\phi_{1}$ as follows. If $\phi \notin G^{+}$, let $\phi_{1}=\phi$. Otherwise, let $\psi_{1}=\phi \phi_{2}$. Then $\psi_{1} \notin G^{+}$and $\psi_{1}$ interchanges $A$ and $B$. Now let $\phi_{1}$ denote $\psi_{1}$ to an appropriate odd power so that $\operatorname{order}\left(\phi_{1}\right)=2^{n}$. Then as we saw above in the proof of Property (1), $\phi_{1}$ is an involution. Also $\phi_{1} \notin G^{+}$and $\phi_{1}$ interchanges $A$ and $B$.

We see that the involutions $\phi_{1}$ and $\phi_{2}$ commute as follows. By Fact 1, $\pi\left(\phi_{1}\right)$ and $\pi\left(\phi_{2}\right)$ are both reflections about great circles. These two circles intersect in a pair of points $p$ and $q$ which are fixed by both $\pi\left(\phi_{1}\right)$ and $\pi\left(\phi_{2}\right)$. Let $P$ and $Q$ be the fibers projecting down to $p$ and $q$. Since $\phi_{1}$ and $\phi_{2}$ are involutions, $\phi_{1} \phi_{2} \phi_{1} \phi_{2}$ pointwise fixes both $P$ and $Q$. Hence by Smith theory $\phi_{1}$ and $\phi_{2}$ commute, and thus we have proved Property (2).

To prove Property (3), suppose the involution $\phi_{2}$ exists and observe that since $\phi_{2}$ reverses the orientation of $A$ and $B$, for every $g \in J, \phi_{2} g \phi_{2} g$ fixes both $A$ and $B$ pointwise. Hence by Smith theory, for every $g \in J, \phi_{2} g \phi_{2}=$ $g^{-1}$.

Finally, to prove Property (4) we suppose that the involution $\phi_{1}$ exists. Thus by Lemma $3,|J|$ is odd and hence $\left|J_{b}\right|$ is odd. In order to choose appropriate generators for $J$, we start with a generator $\alpha$ of $N$ and some $\beta \in J$ such that $\pi(\beta)$ is a generator for $J_{b}$. Observe that $\pi(\beta)^{2}$ is also a generator of $J_{b}$, since $\left|J_{b}\right|$ is odd.

Case 1: $\phi_{1} \in G^{+}$.

Since no element of $G_{b}^{+}$pointwise fixes $C, G_{b}^{+}$induces an isomorphic group action on $C$. Now $\pi\left(f_{1}\right)$ reflects $C$ and $\pi(\beta)$ rotates $C$. Thus $\pi\left(\phi_{1} \beta^{-1} \phi_{1}\right)=$ $\pi(\beta)$. Let $g_{1}=\beta \phi_{1} \beta^{-1} \phi_{1}$. Then $\pi\left(g_{1}\right)=\pi(\beta)^{2}$ since $\pi\left(\phi_{1} \beta^{-1} \phi_{1}\right)=\pi(\beta)$. Let $g_{2}=\alpha$. Since $\pi(\beta)^{2}$ is a generator for $J_{b}, g_{1}$ and $g_{2}$ generate $J$. Now $\phi_{1} g_{1} \phi_{1}=\phi_{1}\left(\beta \phi_{1} \beta^{-1} \phi_{1}\right) \phi_{1}=\phi_{1} \beta \phi_{1} \beta^{-1}=g_{1}^{-1}$, and by Fact $2, \phi_{1} g_{2} \phi_{1}=g_{2}$

Case 2: $\phi_{1} \notin G^{+}$.

Since $\phi_{1}$ is an involution, $\pi\left(\phi_{1}\right)$ is a reflection by Fact 1 . Now since $\pi\left(\phi_{1}\right)$ interchanges $x$ and $y$, fix $\left(\pi\left(\phi_{1}\right)\right)=C$. Also $\pi\left(\beta \phi_{1} \beta^{-1} \phi_{1}\right) \in G_{b}^{+}$and fixes $C$ pointwise. It follows that $\pi\left(\beta \phi_{1} \beta^{-1} \phi_{1}\right)$ is the identity and hence $\pi\left(\phi_{1}\right)$ and $\pi(\beta)$ commute. Let $g_{2}=\beta \phi_{1} \beta \phi_{1}$. Then $\pi\left(g_{2}\right)=\pi(\beta)^{2}$ is a generator of $J_{b}$. 
Let $g_{1}=\alpha$. Then $g_{1}$ and $g_{2}$ generate $J$. Now $\phi_{1} g_{2} \phi_{1}=\phi_{1}\left(\beta \phi_{1} \beta \phi_{1}\right) \phi_{1}=$ $\left(\phi_{1} \beta \phi_{1}\right) \beta=\beta\left(\phi_{1} \beta \phi_{1}\right)$, since $\beta$ and $\phi_{1} \beta \phi_{1}$ are both elements of $J$ which is abelian by Lemma 4 . Thus $\phi_{1} g_{2} \phi_{1}=g_{2}$. Now by Fact 1 , there are fibers $P$ and $Q$ which are reversed by $\phi_{1}$. Then $\phi_{1} g_{1} \phi_{1} g_{1} \mid P$ and $\phi_{1} g_{1} \phi_{1} g_{1} \mid Q$ are the identity. By Smith theory, $\phi_{1} g_{1} \phi_{1}=g_{1}^{-1}$, and we have proved Property (4).

We make the following observation which we will use throughout the proof of Proposition 2. Let $G_{1}$ be the group generated by $J$ together with an involution $\phi_{1}$ which interchanges $A$ and $B$ (if such an involution exists), and let $G_{2}$ be the group generated by $J$ together with an involution $\phi_{2}$ which reverses the orientation of both $A$ and $B$ (if such an involution exists). Let $T_{i}$ be the group generated by $\phi_{i}$. Then $T_{i} \cong \mathbb{Z}_{2}$ and $T_{i} \cap J=1$. Also for every $j \in J, \phi_{i} j \phi_{i} \in J$, and hence $J$ is normal in $G_{i}$. Now $\phi_{i} j=\left(\phi_{i} j \phi_{i}\right) \phi_{i} \in J T_{i}$, and hence $G_{i}=J T_{i}$. It follows that, for each $i, G_{i}=J \rtimes_{\Psi_{i}} \mathbb{Z}_{2}$, where $\Psi_{i}$ acts on $J$ by conjugation by $\phi_{i}$.

Proposition 2. Let $G$ be a finite subgroup of $S O(4)$ which satisfies the Involution Condition and preserves some Seifert fibration of $S^{3}$. Then $G$ is cyclic, dihedral, or a subgroup of $D_{m} \times D_{m}$ for some odd number $m$.

Proof. If $G=J$, then by Lemma $4, G$ is cyclic or $\mathbb{Z}_{q} \times \mathbb{Z}_{r}$ where $q r$ is odd, and the result is evident. So we assume $G \neq J$. Then $G$ is generated by $J$ together with the involution(s) $\phi_{1}$ and/or $\phi_{2}$ given by Lemma 5 .

Case 1: $\phi_{1}$ exists.

It follows from Lemma 3 that $|J|$ is odd. Let $G_{1}$ be the subgroup generated by $J$ and $\phi_{1}$. Then $G_{1}=J \rtimes_{\Psi_{1}} \mathbb{Z}_{2}$, where $\Psi_{1}$ acts on $J$ by conjugation by $\phi_{1}$. Now let $g_{1}$ and $g_{2}$ be the generators for $J$ given by Lemma 5. Thus $\phi_{1} g_{1} \phi_{1}=g_{1}^{-1}$ and $\phi_{1} g_{2} \phi_{1}=g_{2}$. Let $q=\operatorname{order}\left(g_{1}\right)$ and $r=\operatorname{order}\left(g_{2}\right)$, so that $G_{1}=D_{q} \times \mathbb{Z}_{r}$. Furthermore, if $\phi_{2}$ exists then by Lemma $5, \phi_{2} g_{i} \phi_{2}=g_{i}^{-1}$ and $\phi_{1}$ and $\phi_{2}$ commute. Hence $\left(\phi_{1} \phi_{2}\right) g_{1}\left(\phi_{1} \phi_{2}\right)=g_{1}$ and $\left(\phi_{1} \phi_{2}\right) g_{2}\left(\phi_{1} \phi_{2}\right)=g_{2}^{-1}$. This implies that $G=D_{q} \times D_{r}$, with generators $g_{1}, g_{2}, \phi_{1}$, and $\phi_{1} \phi_{2}$. Now, whether or not $\phi_{2}$ exists, $G$ is a subgroup of $D_{m} \times D_{m}$ where $m=q r$ is odd.

Case 2: $\phi_{1}$ does not exist.

Since $G \neq J, G$ is generated by $J$ together with the involution $\phi_{2}$. By Lemma 5 , for every $g \in J, \phi_{2} g \phi_{2}=g^{-1}$. Also, $G=J \rtimes_{\Psi_{2}} \mathbb{Z}_{2}$, where $\Psi_{2}$ acts on $J$ by conjugation by $\phi_{2}$. If $J$ is cyclic, it follows that $G$ is dihedral. Otherwise, $J=\mathbb{Z}_{q} \times \mathbb{Z}_{r}$ and it follows that $G$ is a subgroup of $D_{q} \times D_{r}$. Since $J$ is not cyclic, $q r$ is odd by Lemma 4 . Hence as in Case $1, G$ is a subgroup of $D_{m} \times D_{m}$ where $m=q r$ is odd.

Finally, we consider finite subgroups of $S O(4)$ which satisfy the Involution Condition and do not preserve any Seifert fibration of $S^{3}$.

Proposition 3. Let $G$ be a finite subgroup of $S O(4)$ which satifies the Involution Condition and preserves no Seifert fibration of $S^{3}$. Then $G$ is isomorphic to either $T$, $O$, or D. 
Proof. Let $\rho: S O(4) \rightarrow S O(3) \times S O(3)$ be the canonical two-to-one homomorphism. Now $\rho \mid G$ is either one-to-one or two-to-one. If $\rho \mid G$ is two-to-one, then $G$ contains the antipodal map, which is a fixed point free involution of $S^{3}$. As this would violate the Involution Condition, $\rho \mid G$ must be one-to-one. It now follows from Dunbar [D] that $G$ is isomorphic to $T, O$, or $D$.

\section{ReAlizability of GROUPS By EMBEDDings of $K_{n}$}

We would like to show that each of the groups listed in Proposition 2 and Proposition 3 is realizable by some embedding of $K_{n}$ in $S^{3}$. To this end, we will use the following result from [FNPT] which tells us that under certain conditions subgroups of realizable groups are realizable by an embedding of the same graph.

Lemma 6 ([FNPT]). Let $\Gamma$ be an embedded 3-connected graph. Let $H$ be a subgroup of $\mathrm{TSG}_{+}(\Gamma)$ which is induced by an isomorphic subgroup $G$ of Diff $_{+}\left(S^{3}\right)$, such that no non-trivial element of $H$ fixes any vertex of $\Gamma$. Then $\Gamma$ can be re-embedded as $\Omega$ such that $H=\mathrm{TSG}_{+}(\Omega)$ and $H$ is still induced by $G$.

Now let $\gamma$ be a graph and let $J$ be a subgroup of $\operatorname{Aut}(\gamma)$. If there is an element of $J$ which interchanges the vertices of some edge $e$, then we will say that $e$ is invertible under $J$. We define the graph $\gamma^{\prime}$ associated with $\gamma$ and $J$ to be the graph obtained from $\gamma$ by adding a vertex in the interior of every edge which is invertible under $J$. Every automorphism of $\gamma$ uniquely determines an automorphism of $\gamma^{\prime}$. Thus we shall abuse notation slightly by treating $J$ as a subgroup of both $\operatorname{Aut}(\gamma)$ and $\operatorname{Aut}\left(\gamma^{\prime}\right)$. Note that no edge of $\gamma^{\prime}$ is invertible under $J$.

Realizability Lemma. Let $\gamma$ be a 3-connected graph, and let $J \leqslant \operatorname{Aut}(\gamma)$ such that no non-trivial element of $J$ fixes any vertex of $\gamma$. Suppose the vertices of $\gamma$ can be embedded as $V$ such that $J$ is induced on $V$ by an isomorphic subgroup $K$ of $\operatorname{Diff}_{+}\left(S^{3}\right)$ which satisfies the Involution Condition. Then for every $H \leq J$ there is an embedding $\Gamma$ of $\gamma$ such that $H=\operatorname{TSG}_{+}(\Gamma)$ and $H$ is induced by an isomorphic subgroup of $K$.

Proof. By hypothesis, $J$ is induced on $V$ by a group $K \leq \operatorname{Diff}_{+}\left(S^{3}\right)$ such that $K \cong J$ and $K$ satisfies the Involution Condition. Let $H \leq J$. Then $H$ is induced on $V$ by some group $G \leq K$ such that $G \cong H$ and $G$ satisfies the Involution Condition. Since $G \cong H$ is finite and every element of $G$ induces some element of $H$, for every $a \in H$ there is a unique $g_{a} \in G$ such that $g_{a}$ induces $a$ on $V$. Hence $G=\left\{g_{a} \mid a \in H\right\}$.

Let $Y$ denote the union of the fixed point sets of all of the non-trivial elements of $G$. Since $G$ is a finite subgroup of $\operatorname{Diff}_{+}\left(S^{3}\right), Y$ is a union of finitely many simple closed curves whose pairwise intersection consists of finitely many points. Let $\gamma^{\prime}$ denote the graph associated with $\gamma$ and $H$. We will find an embedding $\Gamma^{\prime}$ of $\gamma^{\prime}$ such that $H=T S G_{+}\left(\Gamma^{\prime}\right)$ and $H$ is 
induced by $G$. First observe that since no non-trivial element of $H$ fixes any vertex of $\gamma$, no non-trivial element of $H$ leaves any edge of $\gamma^{\prime}$ setwise invariant; and each vertex of $\gamma^{\prime}-\gamma$ is fixed by some involution of $H$. In order to embed the vertices of $\gamma^{\prime}-\gamma$, we start by considering those simple closed curves in $S^{3}$ which are the fixed point set of some involution of $G$. Note that by the Involution Condition, for each involution $g \in G$, fix $(g)$ is a simple closed curve which is not fixed by any other non-trivial element of $G$. Let $\left\{f_{1}, \ldots, f_{r}\right\}$ be a set of simple closed curves consisting of one representative from the orbit of the fixed point set of each involution in $G$. For each $i$, we let $a_{i}$ denote the involution in $H$, such that $f_{i}=\operatorname{fix}\left(g_{a_{i}}\right)$. Now let $\left\{\epsilon_{i 1}, \ldots, \epsilon_{i r_{i}}\right\}$ be a (possibly empty) set consisting of one representative from each orbit of the collection of edges of $\gamma$ which are inverted by $a_{i}$. Then $\epsilon_{i j}$ and $\epsilon_{k l}$ have disjoint orbits unless both $i=k$ and $j=l$. Also, $\epsilon_{i j}$ is not setwise invariant under any non-trivial element of $H$ other than $a_{i}$. Now since $G$ satisfies the Involution Condition, we can choose a set of distinct points $\left\{z_{i 1}, \ldots, z_{i r_{i}}\right\} \subset f_{i}$ whose orbits are disjoint and none of which is fixed by any non-trivial element of $G$ other than $g_{a_{i}}$.

We embed each vertex $v \in \gamma^{\prime}-\gamma$ as follows. Since $v$ must be on some invertible edge $\epsilon$ of $\gamma$, there exists $a \in H$, and $i$ and $j$, such that $\epsilon=a\left(\epsilon_{i j}\right)$. If we also have $\epsilon=b\left(\epsilon_{k l}\right)$, then $i=k, j=l$ and either $a=b$ or $a b^{-1}$ is the involution $a_{i}$. Thus $g_{a b^{-1}}$ fixes $f_{i}$, and hence $g_{a}\left(z_{i j}\right)=g_{b}\left(z_{i j}\right)$. Thus we can unambiguously embed $v$ as $g_{a}\left(z_{i j}\right)$. Let $V^{\prime}$ denote $V$ together with all of the vertices of $\gamma^{\prime}-\gamma$ embedded in this way. Then $V^{\prime}$ is an embedding of the vertices of $\gamma^{\prime}$, and $G$ induces $H$ on $V^{\prime}$.

Next we will embed the edges of $\gamma^{\prime}$. Let $\left\{e_{1}, \ldots, e_{n}\right\}$ be a set consisting of one representative from each orbit of the edges of $\gamma^{\prime}$. For each $i$, let $v_{i}$ and $w_{i}$ be the vertices of $e_{i}$ and let $x_{i}$ and $y_{i}$ be the corresponding embedded vertices in $V^{\prime}$. For each $i$, let $\alpha_{i}$ be a path in $S^{3}$ from $x_{i}$ to $y_{i}$ whose interior is disjoint from $V \cup Y$.

Let $\pi: S^{3} \rightarrow S^{3} / G$ denote the quotient map. Then $\pi$ is a branched covering map, and the quotient space $Q=S^{3} / G$ is a 3 -manifold. For each $i$, let $\alpha_{i}^{\prime}=\pi \circ \alpha_{i}$. Then $\alpha_{i}^{\prime}$ is a path or loop in $Q$ from $\pi\left(x_{i}\right)$ to $\pi\left(y_{i}\right)$. Using general position in $Q$, we can homotop each $\alpha_{i}^{\prime}$ rel its endpoints to a simple path or loop $\rho_{i}^{\prime}$ such that the interiors of the $\rho_{i}^{\prime}(I)$ are pairwise disjoint, and are each disjoint from $\pi(V \cup Y)$. For each $i$, let $\rho_{i}$ be the lift of $\rho_{i}^{\prime}$ to $S^{3}$ beginning at $x_{i}$. Since $\rho_{i}^{\prime}=\pi \circ \rho_{i}$ is one-to-one except possibly on the set $\{0,1\}, \rho_{i}$ must also be one-to-one except possibly on the set $\{0,1\}$. Also since $\rho_{i}^{\prime}$ is homotopic rel its endpoints to $\alpha_{i}^{\prime}, \rho_{i}$ is homotopic rel its endpoints to $\alpha_{i}$. Thus $\rho_{i}$ is a simple path from $x_{i}$ to $y_{i}$.

Let $R=\left\{\rho_{1}(I), \ldots, \rho_{n}(I)\right\}$. Now suppose that for some $g_{a} \in G$ and some $i$ and $j, g_{a}\left(\rho_{i}(I)\right)$ and $\rho_{j}(I)$ have the same endpoints. Thus $g_{a}\left(\left\{x_{i}, y_{i}\right\}\right)=$ $\left\{x_{j}, y_{j}\right\}$. It follows that $a\left(\left\{v_{i}, w_{i}\right\}\right)=\left\{v_{j}, w_{j}\right\}$. Hence $e_{i}$ and $e_{j}$ are in the same orbit under $H$, and hence $i=j$. But, since no non-trivial element of $H$ fixes any edge of $\gamma^{\prime}, g_{a}$ must be the identity. Thus the orbit of $R$ under $G$ contains at most one path between a given pair of vertices. 
For each $i$, we embed $e_{i}$ as $\rho_{i}(I)$. Now for an arbitrary edge $e$ of $\gamma^{\prime}$ there is a unique $a \in H$ and $i$ such that $e=a\left(e_{i}\right)$. Thus the vertices of $e$ are $a\left(v_{i}\right)$ and $a\left(w_{i}\right)$, and they have been embedded as $g_{a}\left(x_{i}\right)$ and $g_{a}\left(y_{i}\right)$. We embed $e$ as the unique arc, $g_{a}\left(\rho_{i}(I)\right)$ in the orbit of $R$, which has vertices $g_{a}\left(x_{i}\right)$ and $g_{a}\left(y_{i}\right)$.

Let $\Gamma^{\prime}$ consist of the vertices $V^{\prime}$ together with the embedded edges described above. Observe that $\Gamma^{\prime}$ is setwise invariant under $G$. Also by our choice of the $\rho_{i}^{\prime}$ in $Q$, the orbit of each $\operatorname{int}\left(\rho_{i}(I)\right)$ under $G$ must be disjoint from $V^{\prime}$ and $g_{a}\left(\rho_{i}(I)\right)$ and $g_{b}\left(\rho_{j}(I)\right)$ must have disjoint interiors unless $a=b$ and $i=j$. Hence $\Gamma^{\prime}$ is an embedded graph with underlying abstract graph $\gamma^{\prime}$.

Let $\Gamma$ be the embedded graph obtained from $\Gamma^{\prime}$ by omitting those vertices which are not in $V$. Then $\Gamma$ has underlying abstract graph $\gamma$ and $G$ induces $H$ on $\Gamma$. Now by Lemma $6, \Gamma$ can be re-embedded as $\Omega$ such that $H=$ $\mathrm{TSG}_{+}(\Omega)$ and $H$ is still induced by $G$.

We use the Realizability Lemma to obtain our desired embeddings in the next two propositions.

Proposition 4. Let $H$ be a group which is cyclic, dihedral, or a subgroup of $D_{m} \times D_{m}$ for some odd number $m$. Then there is an embedding $\Gamma$ of some $K_{n}$ such that $H=\mathrm{TSG}_{+}(\Gamma)$ and $H$ is induced by an isomorphic subgroup of $S O(4)$.

Proof. Case 1: $H$ is a subgroup of $D_{m}$, where $m$ is even.

We will find an embedding of $K_{2 m}$ in $S^{3}$ such that $\operatorname{TSG}_{+}(\Gamma)=H$. Let $F_{1} \cup F_{2}$ be a Hopf link and let $g$ be a glide rotation of order $m$ which rotates about $F_{1}$ by $2 \pi / m$ while rotating about $F_{2}$ by $4 \pi / m$. Let $f$ be a rotation by $\pi$ around a circle $S$ which intersects each of $F_{1}$ and $F_{2}$ orthogonally in two points. Now let $G$ be the subgroup of $S O(4)$ generated by $g$ and $f$. It is not hard to check that $G \cong D_{m}$.

Observe that every involution in $G$ other than $g^{m / 2}$ has the form $g^{i} f$ for some non-negative $i<m$. Furthermore, fix $\left(g^{m / 2}\right)=F_{2}$, and for each $i<m$, fix $\left(g^{i} f\right)$ is a circle which intersects $F_{1}$ orthogonally in a distinct pair of points. Also, for every non-trivial $g \in G$, fix $(g)$ is non-empty if and only if $g$ is an involution. It follows that $G$ satisfies the Involution Condition.

Let $v_{1}$ be a point on $F_{1}-\bigcup_{i<m} \operatorname{fix}\left(g^{i} f\right)$. Then the orbit of $v_{1}$ under $G$ is contained in $F_{1}$. Also since $v_{1}$ is not fixed by any non-trivial element of $G$, the orbit of $v_{1}$ contains $2 m$ points. We denote the orbit of $v_{1}$ by $\left\{v_{1}, \ldots, v_{2 m}\right\}$. Let $J$ denote the subgroup of $\operatorname{Aut}\left(K_{2 m}\right)$ which $G$ induces on $\left\{v_{1}, \ldots, v_{2 m}\right\}$. Then $J \cong G \cong D_{m}$ and no non-trivial element of $J$ fixes any embedded vertex of $K_{2 m}$.

Since $H \leq J$, we can apply the Realizability Lemma to $H$ to conclude that there is an embedding $\Gamma$ of $K_{2 m}$ such that $H=\mathrm{TSG}_{+}(\Gamma)$ and $H$ is induced by an isomorphic subgroup of $G$.

Case 2: $H$ is a subgroup of $D_{m} \times D_{m}$ where $m$ is odd. 
We will find an embedding $\Gamma$ of $K_{4 m^{2}}$ in $S^{3}$ such that $\operatorname{TSG}_{+}(\Gamma)=H$. Consider a torus $T$ in $S^{3}$ which bounds two isometric solid tori. Let $\varphi$ denote the glide rotation of $S^{3}$ which rotates each $(1,1)$ curve on $T$ along itself by $2 \pi / m$, and let $\psi$ denote the glide rotation which rotates each $(-1,1)$ curve on $T$ along itself by $2 \pi / m$. Let $f$ be a rotation by $\pi$ about a particular $(1,1)$ curve $X$ and let $g$ be a rotation by $\pi$ about a particular $(-1,1)$ curve $Y$. Let $G$ be the subgroup of $S O(4)$ generated by $\phi, \psi, f$ and $g$.

The following two observations are not hard to show:

1. $\varphi \psi=\psi \varphi, f g=g f, f \varphi=\varphi f$, and $g \psi=\psi g$

2. $f \psi f=\psi^{-1}$ and $g \varphi g=\varphi^{-1}$

Using these observations we can deduce that $G=D_{m} \times D_{m}$. Furthermore, since $m$ is odd, every involution in $G$ has the form $\psi^{j} f, \phi^{i} g$, or $\phi^{i} \psi^{j} f g$ for some $i, j<m$. Now for each $i$ and $j, \operatorname{fix}\left(\psi^{j} f\right)$ is a distinct $(1,1)$ curve on $T$, $\operatorname{fix}\left(\phi^{i} g\right)$ is a distinct $(-1,1)$ curve on $T$, and $\operatorname{fix}\left(\phi^{i} \psi^{j} f g\right)$ is a distinct circle which meets $T$ orthogonally in four points. Also, for every non-trivial $j \in G$, $\operatorname{fix}(j)$ is non-empty if and only if $j$ is an involution. It follows that $G$ satisfies the Involution Condition.

Let $A=\bigcup_{j<m}$ fix $\left(\psi^{j} f\right)$ and let $B=\bigcup_{i<m}$ fix $\left(\phi^{i} g\right)$. Observe that the sets $A$ and $B$ each contain $m$ curves, and each curve in $A$ meets each curve in $B$ in two points. Thus $T-(A \cup B)$ consists of $2 m^{2}$ squares. The collection of corners and centers of the closures of these squares are precisely the $4 \mathrm{~m}^{2}$ points where $\bigcup_{i, j<m} \operatorname{fix}\left(\phi^{i} \psi^{j} f g\right)$ meets $T$. Observe that for any given square $S$ in $T-(A \cup B)$, all of the squares in $T-(A \cup B)$ are in its orbit; and the unique non-trivial element of $G$ which takes $S$ to itself is the involution of the form $\phi^{i} \psi^{j} f g$ which fixes the center point of $S$.

Let $v_{1}$ be a point on $T-(A \cup B)$ which is not in the center of a square. Then the orbit of $v_{1}$ under $G$ consists of two points in each of the $2 \mathrm{~m}^{2}$ squares of $T-(A \cup B)$. Let $\left\{v_{1}, \ldots, v_{4 m^{2}}\right\}$ denote the orbit of $v_{1}$ under $G$, and let $J$ denote the subgroup of $\operatorname{Aut}\left(K_{4 m^{2}}\right)$ which $G$ induces on $\left\{v_{1}, \ldots, v_{4 m^{2}}\right\}$. Then $J \cong G \cong D_{m} \times D_{m}$ and no non-trivial element of $J$ fixes any embedded vertex of $K_{4 m^{2}}$.

Since $H \leq J$, we can apply the Realizability Lemma to $H$ to conclude that there is an embedding $\Gamma$ of $K_{4 m^{2}}$ such that $H=\operatorname{TSG}_{+}(\Gamma)$ and $H$ is induced by an isomorphic subgroup of $G$.

Proposition 5. Let $H$ be one of the groups $T, O$, or $D$. Then there is an embedding $\Gamma$ of some $K_{n}$ such that $H=\mathrm{TSG}_{+}(\Gamma)$ and $H$ is induced by an isomorphic subgroup of $S O(4)$.

Proof. First let $\Lambda$ be the 1-skeleton of a symmetric 4-simplex embedded in $\mathbb{R}^{4}$ with the origin at its center, and let $\Gamma$ be the radial projection of $\Lambda$ to $S^{3}$. Then $\Gamma$ is an embedding of $K_{5}$ in $S^{3}$. Let $G$ denote the group of orientation preserving isometries of $\Lambda$ in $\mathbb{R}^{4}$. It is not hard to show that $G$ is isomorphic to the alternating group $A_{5}, G$ induces an isomorphic action on $\left(S^{3}, \Gamma\right)$, and $\mathrm{TSG}_{+}(\Gamma) \cong A_{5}$. It is well known that $A_{5} \cong D$. 
Next we consider the octahedral group $O$. We will create a subgroup $G$ of $S O(4)$ which is isomorphic to $O$ but is not the group of symmetries of a regular octahedron or cube. We begin by radially projecting a cube centered at the origin in $S^{3}$ to a 2-complex $C$ which is contained in the unit 2 -sphere. Let $f$ be the rotation by $2 \pi / 3$ about an axis which runs through two antipodal vertices of $C$. Let $S=a \cup d \cup b \cup e$ where $a$ is a diagonal of one face of $C, b$ is the parallel diagonal of the opposite face of $C$, and $d$ and $e$ are the edges of $C$ which connect the endpoints of $a$ and $b$. Let $g$ be the rotation by $\pi$ about $S$. Finally, let $h$ be the glide rotation which rotates by $\pi / 2$ about an axis $X$ going through the center of two opposite faces of $C$, while rotating about a disjoint equator by $\pi$. We can choose $f, g$, and $h$ so that $g h f$ is the identity (see Figure 1). Let $G$ be the subgroup of $S O(4)$ which is generated by $f, g$, and $h$. By inspection, we see that the group $G$ has 24 elements and every non-trivial element of $G$ either has order 2 , 3, or 4 and is conjugate to $g, f$, or $h$ respectively. It is not hard to check that $G \cong O$.

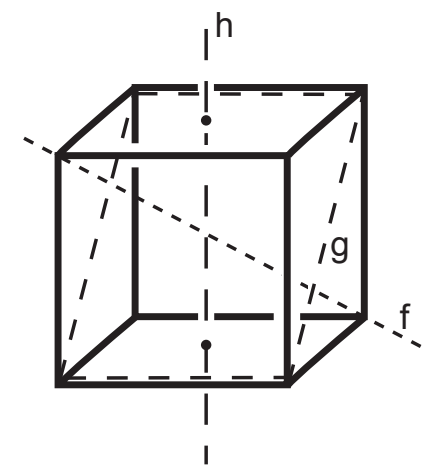

Figure 1. The generators of $G$

We consider the fixed point sets of the elements of $G$ as follows. Let $j \in G$. If $j$ is an involution, then either $\operatorname{fix}(j)$ is a circle which meets $C$ in the centers of two opposite faces, or $\operatorname{fix}(j)$ is made up of two parallel diagonals on opposite faces of $C$ together with two edges connecting the endpoints of these diagonals. If $j$ has order 3 , then $\operatorname{fix}(j)$ is an axis which runs through antipodal vertices. Finally, if $j$ has order 4 , then $\operatorname{fix}(j)$ is empty. It follows from these observations that $G$ satisfies the Involution Condition.

Observe that $\left(\bigcup_{j \in J} \operatorname{fix}(j)\right) \cap C$ is the union of all the edges of $C$ together with the diagonals of all of the faces of $C$. We shall construct an embedding of $K_{24}$ as follows. Let $v_{1}$ be a point on $C$ which does not lie on any edge 
or any diagonal of any face of $C$. Then the orbit of $v_{1}$ under $G$ contains 24 points, which we denote by $\left\{v_{1}, \ldots, v_{24}\right\}$. Let $H$ denote the subgroup of $\operatorname{Aut}\left(K_{24}\right)$ which $G$ induces on $\left\{v_{1}, \ldots, v_{24}\right\}$. Then $H \cong G$ and no nontrivial element of $H$ fixes any embedded vertex of $K_{24}$. Thus we can apply the Realizability Lemma to get an embedding $\Gamma$ of $K_{24}$ such that $H=$ $O=\mathrm{TSG}_{+}(\Gamma)$, and $H$ is induced by $G$. Furthermore, since $T \leq O$, the Realizability Lemma also gives us an embedding $\Gamma$ of $K_{24}$ such that $T=$ $\mathrm{TSG}_{+}(\Gamma)$, and $T$ is induced by an isomorphic subgroup of $G$

\section{Conclusion}

We will now put together our five Propositions to complete our characterization of those groups which are $\operatorname{TSG}_{+}(\Gamma)$ for some embedding $\Gamma$ of a complete graph.

Theorem 1. A finite group $H$ is $\mathrm{TSG}_{+}(\Gamma)$ for an embedding $\Gamma$ of some $K_{n}$ if and only if $H$ is isomorphic to a finite subgroup of either $S O(3)$ or $D_{m} \times D_{m}$ for some odd $m$.

Proof. First note that the finite subgroups of $S O(3)$ are precisely the finite cyclic groups, the dihedral groups, and $T, O$, and $D$.

Suppose that there is an embedding $\Gamma$ of some $K_{n}$ with $H=\mathrm{TSG}_{+}(\Gamma)$. By Proposition 1, either $H$ is cyclic of odd order or there exists an embedding $\Gamma^{\prime}$ of $K_{n}$ such that $H$ is induced on $\Gamma^{\prime}$ by an isomorphic subgroup $G$ of $S O(4)$. If $H$ is cyclic we are done. So we assume that $H$ is induced by an isomorphic subgroup $G$ of $S O(4)$. Then, by Lemma 2, $G$ satisfies the Involution Condition. If $G$ preserves some Seifert fibration of $S^{3}$, then by Proposition 2, $G$ is either cyclic, dihedral, or a subgroup of $D_{m} \times D_{m}$ for some odd number $m$. So in this case we are done. On the other hand, if $G$ preserves no Seifert fibration of $S^{3}$, then by Proposition 3, $G$ is isomorphic to $T, O$, or $D$. So again we are done.

In order to prove the converse, first suppose that $H$ is cyclic, dihedral, or a subgroup of $D_{m} \times D_{m}$, for some odd number $m$. Then by Proposition 4, there is an embedding $\Gamma$ of some $K_{n}$ such that $H=\mathrm{TSG}_{+}(\Gamma)$. On the other hand, if $H$ is $T, O$, or $D$, then by Proposition 5, there is an embedding $\Gamma$ of some $K_{n}$ such that $H=\mathrm{TSG}_{+}(\Gamma)$.

Finally, observe that the following result follows immediately from Theorem 1 together with Propositions 4 and 5.

Corollary 1. Let $\Omega$ be an embedding of some complete graph with $H=$ $\mathrm{TSG}_{+}(\Omega)$. Then there exists an embedding $\Gamma$ of some (possibly different) complete graph such that $H=\mathrm{TSG}_{+}(\Gamma)$ and $H$ is induced by an isomorphic subgroup of $S O(4)$.

\section{REFERENCES}

[BLP] M. Boileau, B. Leeb, and J. Porti : Uniformization of small 3-orbifolds, C. R. Acad. Sci. Paris Sér. I Math. 332 (2001), 57-62. 
[BP] M. Boileau and J. Porti : Geometrization of 3-orbifolds of cyclic type, Astérisque 272, SMF Publications, Paris, 2001.

[CHK] D. Cooper, C. Hodgson, and S. Kerckhoff : Three-dimensional orbifolds and cone-manifolds, Mathematical Society of Japan Memoirs 5, Tokyo, 2000.

[D] W. D. Dunbar : Nonfibering spherical 3-orbifolds, Trans. Amer. Math. Soc. 341 (1994), 121-142.

[F] E. Flapan : Symmetries of Möbius ladders, Math. Ann. 283 (1989), 271-283.

[FNPT] E. Flapan, R. Naimi, J. Pommersheim, and H. Tamvakis : Topological symmetry groups of embedded graphs in the 3-sphere, preprint (2003).

[O] P. Orlik: Seifert Manifolds, Lecture Notes in Mathematics, Vol. 291. SpringerVerlag, Berlin-New York, 1972.

[N] C. Noda: The topological symmetry group of a canonically embedded complete graph in $S^{3}$, Tokyo J. Math. 20 (1997), 45-50.

[Si] J. Simon : A topological approach to the stereochemistry of nonrigid molecules, Graph theory and topology in chemistry (Athens, GA, 1987), Stud. Phys. Theoret. Chem. 51, 43-75, Elsevier, Amsterdam, 1987.

[Sm] P. A. Smith : Transformations of finite period II, Annals of Math. 40 (1939), 690-711.

Department of Mathematics, Pomona College, Claremont, CA 91711, USA

E-mail address: eflapan@pomona.edu

Department of Mathematics, Occidental College, Los Angeles, CA 90041, USA

E-mail address: rnaimi@oxy.edu

Department of Mathematics, Brandeis University - MS 050, P. O. Box 9110, WALTHAM, MA 02454-9110, USA

E-mail address: harryt@brandeis.edu 